\title{
Téoros
}

Revue de recherche en tourisme

\section{À propos de paysage culturel}

Le patrimoine architectural religieux, une offre distinctive au Québec?

\section{Luc Noppen et Lucie K. Morisset}

Volume 16, numéro 2, été 1997

Tourisme et religion

URI : https://id.erudit.org/iderudit/1074574ar

DOI : https://doi.org/10.7202/1074574ar

Aller au sommaire du numéro

Éditeur(s)

Université du Québec à Montréal

ISSN

0712-8657 (imprimé)

1923-2705 (numérique)

Découvrir la revue

Citer cet article

Noppen, L. \& Morisset, L. K. (1997). À propos de paysage culturel : le patrimoine architectural religieux, une offre distinctive au Québec ? Téoros, 16(2), 14-20. https://doi.org/10.7202/1074574ar d'utilisation que vous pouvez consulter en ligne.

https://apropos.erudit.org/fr/usagers/politique-dutilisation/ 


\section{$+\ldots . .$.}

\section{À propos de paysage culturel}

\section{LE PATRIMOINE ARCHITECTURAL RELIGIEUX, UNE OFFRE DISTINCTIVE AU QUÉBEC ? ${ }^{1}$}

Luc Noppen, professeur et chercheur École d'architecture et CELAT ${ }^{2}$ - Université Laval, Québec

Lucie K. Morisset, professeure et chercheure Département d'études urbaines et touristiques - UQAM et CELAT - Université Laval

La désaffectation de la pratique religieuse au Québec - comme ailleurs - pose avec plus d'acuité que jamais l'épineux problème de la conservation du patrimoine, en l'occurrence des églises que la collectivité, par dela le culte, juge nécessaire de conserver. Or la survie, voire la seule existence de ce patrimoine, ressortit, pour une grande part, à l'offre touristique qu'il constitue potentiellement, soit au regard des communautés locales et régionales, soit au regard des visiteurs internationaux. L'objectif de cet article est d'explorer ce "patrimoine sà la recherche des ingrédients qui, justement, valideraient la participation des églises au corpus des * mises en tourisme $\$$ québécoisest.

En 1996, en page couverture d'un livre sur les églises de la ville de Québec, nous reprenions ce slogan que nous avions imaginé dans le cadre d'une étude sur les lieux de culte : $\$$ nos églises sont nos chăteaux $m^{5}$.

Les monuments religieux sont certes \& à la mode * ces jours-ci ; à vrai dire, il ne se passe une semaine sans que l'un ou l'autre fasse la manchette de quelque journal. Ce qui n'explique pourtant pas que cette phrase fort simple « nos églises sont nos châteaux $\%$ ait, depuis, contaminé l'imaginaire collectif. Utilisée par plusieurs, à Québec ou ailleurs, la métaphore a apparemment « colle $"$ au sujet. Or, si les associations de ce genre, généralement fortuites, ne méritent guère d'être explorées plus avant, il semble que celleci est digne de quelque attention, à tout le moins en ce qui concerne les rapports entre le tourisme et les églises
Sans doute convient-il de postuler quelques prémisses au départ de cette exploration, dont, a priori, qu' une part de l'intérêt actuel pour les églises, communément désignées * patrimoine $*$, tient d'un contenu culturel qu'on leur impute. Puis, il faut envisager que cette teneur * patrimoniale $*$ ressortisse probablement aux mêmes champs d'intérêt que les autres attractions dites * culturelles $*$ vers lesquelles convergent les touristes : c'est dire que le « patrimoine architectural religieux $*$ recèlerait un potentiel économique. On pourrait vendre des églises sur le marché des attractions.

C'est de ce point de vue que la popularité de cette phrase \& nos églises sont nos châteaux s s'avère une introduction parfaite à l'analyse des possibles rapports entre le tourisme au Québec et les églises au Québec. En effet, par delă la configuration matérielle et l'histoire, il apparait que c'est la vocation connotée par la métaphore qui justifie sa diffusion, à l'heure où, justement, les églises sont de moins en moins des églises, du moins au sens cultuel de la dénotation?

Tant dans l'imaginaire collectif québécois que dans la réalité, les églises sont effectivement, ou peuvent être, des châteaux. Le slogan, évidemment, réfere au modẻle français des châteaux de la Loire, très connus, très visités, et dont la notoriété continue de croître au fil d'une mise en tourisme soigneusement orchestrée. En ce sens, "les églises sont nos châteaux * signifie qu'elles possèdent cette même valeur préminente que les chăteaux européens pour certaines régions ou pour des pays entiers. Comme ce que le Louvre est à Paris, comme ce que les chateaux sont à la France ${ }^{x}$, les églises seraient au Québec les monuments ultimes d'un paysage distinctif, à la fois symboles et paysages".

Nous avons déjà discuté du processus conduisant à un tel statut, que nous présupposons ici (Noppen et Morisset, 1997b) ; inutile donc de revenir sur la construction de la notoriété des églises et leur progressive inscription dans l'imaginaire collectif, pas plus que sur celle des châteaux de la Loire. C'est plutôt en aval de ce processus, au moment où l'on déclare que " les églises sont nos châteaux $*$, que nous situons la réflexion amorcée dans le cadre de cet article. A l'enseigne du thème * tourisme et religion w, il nous apparaît en effet que les églises sont les porteuses tacites d'une ressource culturelle que l'inconscient collectif leur attribue spontanément. En d'autres mots, sans que l'on sache vrai- 
ment pourquol, et sans que cette valeur soit véritablement exploitée (comme l'est, au contraire, celle des châteaux français), la collectivité québécoise confêre aux églises le rôle de monuments fédérateurs du paysage national ${ }^{10}$. Adopter ce $\alpha$ nos églises sont nos châteaux \% c'est admettre, en fait, que les églises sont au centre $d^{4} u n e$ offre touristique potentielle du Québec. Or, à l'heure où les réflexions sur l'offre touristique québécoise commencent à peine à aborder l'objet qui, en bout du compte, sera offert ${ }^{14}$, cette option se révêle d'un intérêt certain : non seulement les églises - comme les châteaux le furent d'ailleurs - sont-elles de plus en plus en quête de nouvelles fonctions, mais le Québec est, quant à lui, en quête d'une offre culturelle digne de mention ${ }^{12}$. Bref, ces églises - $*$ nos châteaux - nous paraissent détenir la clế d'une réconciliation du tourisme et du patrimoine, et, dans la foulée, d'une offre touristique de la culture québécoise.

$$
\text { 事来 }
$$

Il apparaît donc qu'un certain nombre de monuments du patrimoine architectural religieux, en dépit de la disparition de leur fonction originelle, méritent d'être conservés et attendent les moyens nécessaires à cette fin. Il apparaît, d'autre part, que le tourisme, possible sustentateur, y trouverait les ingrédients d'une offre originale, distinctive de celles qui encadreraient l'inscription du Québec dans le paysage culturel occidental ${ }^{13}$.

\section{L'ÉGLISE AU CENTRE DU PAYSAGE QUÉBÉCOIS}

D'entrée de jeu, il faut spécifier que la prééminence des églises dans l'image des villages et des villes n'est peut-être pas tant l'apanage du Québec que celui du Canada français. On peut, à vrai dire, reconnaitre les paysages des communautés francophones partout au Canada et en Nouvelle-Angleterre, autour des églises qui les fédèrent ; il paraît exister une a figure connue $*$ de l'église qui pourrait être partiellement expliquée par la communauté des usages dans la $\alpha$ diaspora $\%$ francophone. Comme serait née une a maison québécoise que certains se sont plu à identifier jusqu"en NouvelleAngleterre, il existerait une « église québecoise $m$ dont la diffusion, via les traditions constructives et cultuelles émigrées, aurait fini par consacrer les modèles exemplaires. Dans tous les cas, par exemple, en ce qui concerne les maisons rurales, il faut constater que tous les visiteurs de la province, Québécois et autres, gardent le sentiment diffus d'une originalitế ancrée aux églises, comme les visiteurs de la France ont l'instinct d'une spécificité de ses châteaux.

L'omniprésence de l'église dans cette définition imaginaire du paysage construit québécois ne peut toutefois pas ressortir au nombre total des églises construites sur le territoire concerné. Quoiqu'en dise la tradition orale, les églises ne sont pas plus nombreuses au Québec qu'ailleurs, ni au total, ni dans chacun des villages. Ailleurs, en France par exemple, il n'est pas rare de compter deux ou trois églises dans un petit village, alors qu'au Québec, on n'en compterait qu'une dans un village similaire. Toutefois tous s'accordent pour dire que les églises du Québec sont plus présentes au sein des paysages concernés que les églises de France. Cette présence accrue s'expliquerait-elle par une sorte de rapport privilégié au paysage environnant qui, tel un écrin, * monumentaliserait * cette présence pardelà la réalité matérielle de chaque église ?

En effet, il existe un certain nombre de dispositions, spécifiques au Canada français parce qu'elles s'y sont superposées dans l'histoire (et ont été préservées), qui définissent, d'une part, une relation particulière entre la structure (imaginaire et concrete) d'un village et, d'autre part, l'église qui y est bătie. Ce qui revient à dire qu'il existe un paysage culturel, abstrait, qui surdétermine le paysage concret et l'église y représente le point d'atterrissage.

Ce rapport au paysage, évidemment, prend surtout corps dans les églises catholiques, principales détentrices de cette * figure connue définitoire des communautés francophones au Canada français. Il faut dire que les églises catholiques sont ici plus nombreuses que les lieux de culte d'autres dénominations (la ville de Québec, par exemple, compte actuellement quelque 68 églises catholiques, sur un total de 98 lieux de culte ${ }^{14}$ ). Comme le prescrit leur nature, dans l'histoire, les églises catholiques sont aussi de dimensions et d'apparence plus monumentale que les églises de plusieurs autres dénominations. Mais, surtout, les églises catholiques, par rapport à celles d'autres cultes et par rapport aux bâtiments d'autres fonctions, sont au Québec le siège de l'Église établie — ou à tout le moins le sont restées, jusqu'à récemment.

Comme le voulait la gouverne sous le pouvoir royal français, l'Église catholique, qui s'était implantée en NouvelleFrance, est restée au Canada en dépit de la Conquête britannique qui devait, en principe, entraîner l'établissement d'une autre église, celle d'Angleterre, associée au gouvernement britannique. En effet, l'Église qui, en principe, était gallicane, plutôt de faire place à l'Église anglicane, a continué d'exister dans une communauté parallèle, organisant le culte pour les Canadiens français, tandis que les membres de l'Église d'Angleterre suivaient le culte en anglais. L'existence de l'ancienne Église catholique a finalement été confirmée par l'Acte de Québec (1774), puis par I'Acte constitutionnel (1791), qui ont permis à l'Église des Canadiens français de survivre grâce, notamment, au maintien des lois civiles françaises, garantes du prélèvement de la dîme, mesure sustentatrice de l'Église depuis les débuts de la coloniét.

D'où l'existence, dans un monde où l'association Église-État était naturelle, de deux Églises établies au Canada dont l'une - la catholique des Canadiens français - continua de dominer le paysage. II en résulte parfois quelques anachronismes. Par exemple, Québec fut sans doute la première ville au monde à compter deux cathédrales de dénominations différentes, construites comme telles. Toutefois, l'effet de la coexistence est surtout tangible, dans la géographie du pays aménagé ces trois derniers siècles. Comme la Loi des fabriques l'a con= sacré et selon les exigences de l'Église établie, le territoire québécois a été partagé en paroisses catholiques, organisées autour d'une église catholique, nonobstant les temples d'autres dénominations ${ }^{16}$.

En effet, c'est au centre de ces unités géopolitiques que, naturellement, on entreprit d'implanter les églises, ce qui a pour corollaire l'image selon laquelle la ville ou le village gravite autour de la haute tour du clocher qui, généralement, domine les lieux. La centralité naturelle de l'église dans l'unité paroissiale 


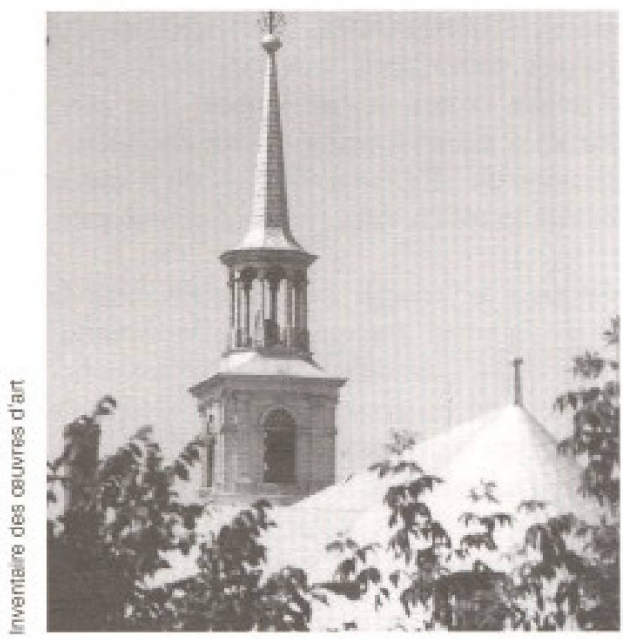

Exemple de ces clochers bien particuliers: clocher de l'église Saint-Pierre-les-Becquets, Thomas Baillairge, architecte.

explique, d'une part, la bipolarité de certaines villes, où la répartition de l'occupation s'est modifice après que l'on eut construit l'église au centre de la paroisse initiale. Jonquière (Saguenay), par exemple, a ainsi été déphasée par l'arrivée du chemin de fer qui étira les limites habitées — celles de la municipalité de paroisse originelle - au-delà de l'aire où l'église, centrale, avait été construite. Dans la plupart des cas toutefois, l'église fut carrément déménagée quand il s'avéra que le centre du village ne s'établirait pas là̀ où on l'escomptait : à Sainte-Anastasic de Lyster ou à Saint-Joachim de Montmorency, par exemple, l'église continua ainsi de s'affirmer pôle unique des etablissements.

Avec des pôles d'une telle force, il n'est pas surprenant que le Québec compte un nombre impressionnant d'enclos paroissiaux - comme il en reste quelques-uns en Bretagne - qui ont été émargés ailleurs par la densification de la ville. Ainsi, cimetière, presbytère, maisons d'écoles, comme à Saint-Mathias de Rouville, à l'Acadie, à Saint-Joseph de Beauce, à Saint-Jean de l'Île-d'Orléans, préservent ces a centres de villages $\$$ consacrés autour de l'église. Il n’est pas surprenant non plus, à l'inverse, que les villages dépouillés de leur église originelle - par un incendie, par exemple - subissent une véritable déconstruction, jusqu"à disparaître. L'unicité de ce clocher est d'ailleurs probante (les villages européens en comptent souvent plusieurs petits) ; le village québécois \& irradie $x$ en quelque sorte de cette tour, sciemment conçue pour être fédératrice. Coiffée d'une flèche qui brille au soleil - alors qu'ailleurs les clochers ne sont souvent que des tours - l'église est le point de référence morphogénétique du développement territorial du Québec. Nulle part l'expression \& esprit de clocher $*$ ne prend d'ailleurs une telle puissance que dans ces villes et ces villages, où tout ce qui est communautaire - on n'a qu'à penser aux Caisses populaires, nées dans les églises et réparties en paroisses émane des églises.

La préminence des églises dans le paysage n'est pas que question de structuration géographique ; elle est de fait aussi question d'image. A la maniềre de ces flèches qui ont confirmé la centralité des églises, quelques dispositions matérielles ont concouru à consacrer leur importance au sein de l'agglomeration. Ainsi, il est probable qu'en aucun pays du monde on ne trouve développée avec autant d'insistance la figure de la maison curiale, compagne éternelle de l'église. Partout au Québec, dans la ville comme à la campagne, les presbytères sont éminemment reconnaissables. La coincidence n'est sîrement pas fortuite si tous les villages connaissent quelque mythologie selon laquelle l'église locale fut construite pour être cathédrale et le presbytère pour être palais épiscopal. La volonté d'une maison imposante, d'un caractère architectural achevé participant forcément à la centralité de l'église, s'explique toutefois assez simplement : la nécessité voulait en effet qu'à l'ouverture d'une paroisse, le premier bâtiment construit soit le presbytère où le curé s'établirait. Le dénuement de la plupart des paroisses exigeait cependant que le presbytère soit à tout le moins suffisamment a architecturé s pour qu'un curé veuille bien s'y établir ; aussi, à défaut de pouvoir financer simultanément le presbytère et l'église, on bâtissait le premier afin que son rez-de-chaussee puisse abriter une chapelle temporaire en attendant. l'argent nécessaire à la construction de l'église. Cette dernière construite, la grande maison du presbytère était alors à l'échelle de la grande église ; l'évêque et sa suite pouvaient désormais accomplir, dans la paroisse ainsi dotée, leur visite annuelle. Ces presbytères, sortes de manoirs élisabéthains, sont forcément devenus les points de référence de la diffusion des courants architecturaux dans les villages. Le soin architectural apporté à leur édifi- cation, posant leur statut de modèle dans le paysage construit des villages, explique enfin l'apparente * appartenance $\%$ des presbytères à la configuration formelle des maisons environnantes, qui en ont tout simplement été inspirées.

Cette image d'appartenance au terroir et donc, à l'inverse, cette impression selon laquelle le village se définit par l'église - est généralement le fait de telles * coincidences $*$ entre $l^{\prime} \mathrm{eg}$ glise et le territoire qui, réinterprétées dans l'imaginaire collectif, ont nourri la prééminence de l'église dans le paysage culturel québécois. Ainsi, de la même façon que l'imaginaire croit les maisons construites selon le climat, parce que leur plus courte façade donne ver's le nord-est (c'est plutôt l'orientation du parcellaire qui dicte cette implantation), les églises semblent pareillement orientées selon le Saint-Laurent qu'elles longent, alors que c'est la tradition chrétienne, voulant le chevet des églises à l'est, qui dicte cette disposition. N'empêche : peu de visiteurs de la vallée du Saint-Laurent Échappent à cette impression selon laquelle les églises, au Québec, suivent le fill de l'eau...

\section{L'ORIGINALITÉ DES ÉGLISES, LA SPÉCIFICITÉ DU PAYSAGE}

Les rapports étroits entre l'église et le paysage quếbếcois étant ainsi établis dans la réalitế et dans l'imaginaire collectif, il n'est guère étonnant que les touristes s'arrêtent aux églises, allant jusqu'à collectionner leurs visites, d'église en église, de village en village. $L$ intime conviction de certains quant à une spécificité de ces monuments, parfois confuse, peut alors prendre ancrage dans les dispositions architecturales de ces églises, effectivement particulières, qu'il convient de remarquer.

La première de ces particularités, et la plus frappante pour l'imaginaire collectif, tient au matériau des constructions. Les églises sont en pierre. Ce qui est bien naturel, compte tenu de la pérennité dont se voyait investie l'architecture, a fortiori les églises, dans le monde méditerranéen. Rome prescrit d'ailleurs qu'une église, pour être consacrée, doit n'avoir aucune dette et être construite en pierre. Mais l'église en pierre reste fort surprenante, et d'autant plus remarquable, dans des vil- 
lages où le développement n'a laissé, encore, que des constructions de bois.

Cependant, si les églises sont en pierre, ici comme ailleurs, elles comportent au Québec une particularité unique : leurs planchers sont de bois. De fait, si l'on peut comparer, par exemple, les intérieurs ornés à ceux d'Amérique latine, ou les retables sculptés à ceux de Bretagne, nulle part ailleurs de tels planchers ont-ils été substitués à la pierre, en raison de la nécessité de dissocier (par un vide, une cave, etc.) la nef du sol (gele l'hiver). Aucune église en pierre n'a cette chaleur quasi domestique que conferent aux églises du Québec les planchers de bois.

Une autre caractéristique ressort de la construction de ces bâtiments, essentiellement constitués d'une enceinte enclosant simplement $l$ 'intérieur ; puisqu'on n'y a pas développé outre mesure la structure basilicale, l'ensemble des cloisons, des porteurs, etc., traditionnellement en pierre, $y$ sont aussi en bois. Mais en raison de l'intếrêt que l'on sait pour les clochers, on a développé, des fausses tours qui, au lieu de porter sur un carré de maçonnerie, sont appuyées à l'intérieur de l'église sur des piliers de bois. Cela aussi est unique : les seules tours maçonnées que compte le Québec sont celles que de nouveaux arrivants (les Britanniques ă l'église Chalmers, à Québec, par exemple) construisirent spontanément, avant de s'accoutumer aux usages locaux, inscrits tant dans les pratiques que dans le paysage.

C'est une telle architecture que l'histoire a dite * de substitution $\%$. D'autres caractéristiques la distinguent, parmi lesquelles les visiteurs européens remarqueront tout particulièrement les voûtes - ou plutôt les fausses voûtes - qui, au lieu d'être en pierre, sont en plâtre, portées sur des cintres. Cette technique, développée pendant la reconstruction de Londres au lendemain de la conflagration de 1666 afin de ne pas poser une trop lourde charge sur des murs affaiblis par l'incendie - prend ici une coloration particulière : en effet, alors que les Britanniques font des plafonds, les Canadiens, héritiers de la Nouvelle-France, construisent plutôt des voûtes, comme celles que la France érigeait en pierre.

II en est ainsi de tous les ingrédients du mariage bien original des églises québé-

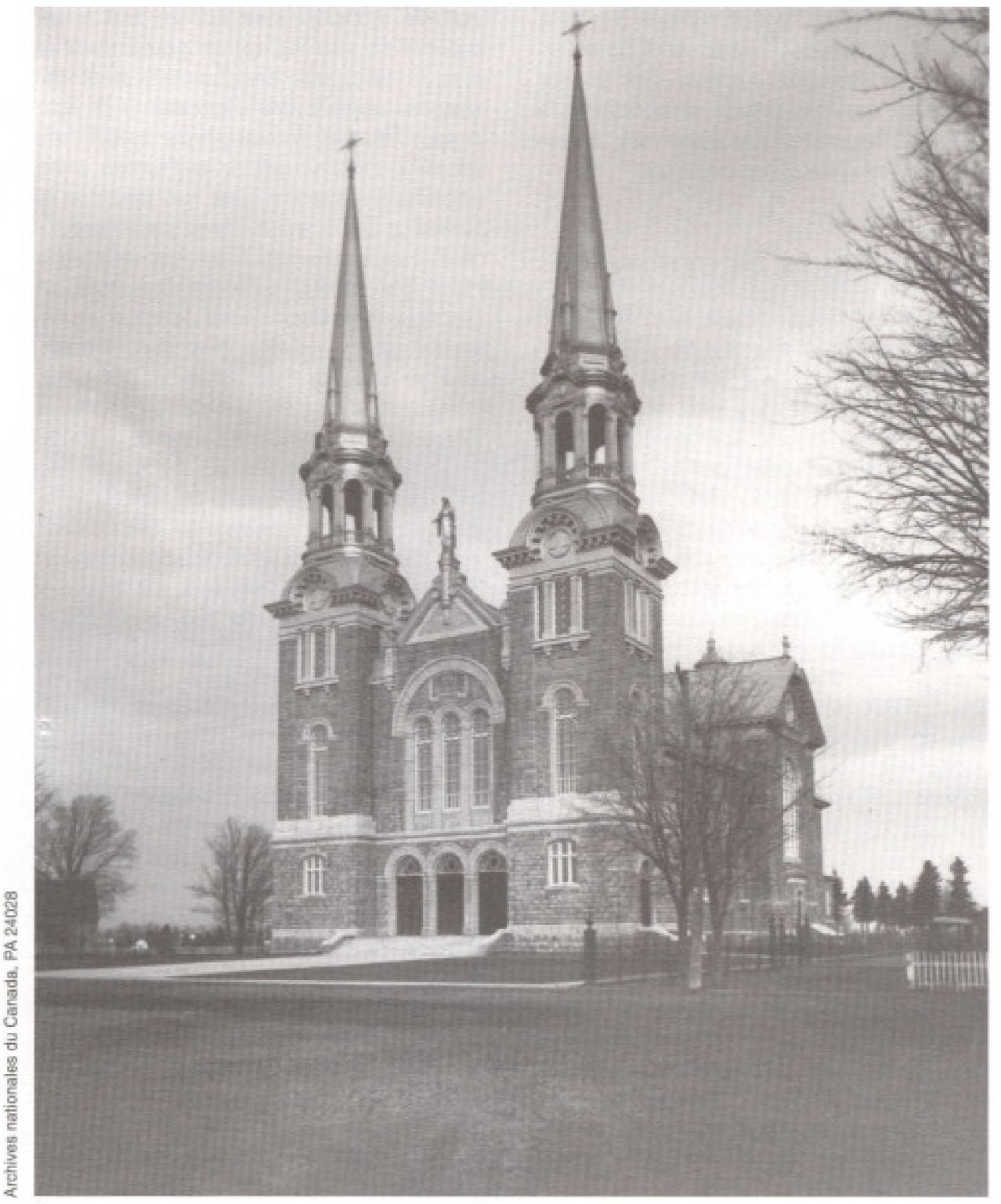

Eglise Notre-Dame-de-l'Annonciation de I'Ancienne-Lorette.

coises et de leur contexte, lui-même né d'un métissage uniqué entre la France, la Grande-Bretagne et l'Amérique du Nord. Solutions de substitution, de compromis, diront certains, ces ingrédients sont surtout le fait d'une spécificité exceptionnelle quí définit un paysage ecclésial incomparable en Occident. On peut encore invoquer a ce chapitre les sacristies ; ces chapelles d'hiver, établies par les Britanniques, $\pi$ 'avaient en effet pas de précédent en Nouvelle-France, alors que les chapelles françaises, ouvertes sur le choeur, ne pouvaient servir un même usage. La préservation du plan en croix latine, issu de la tradition méditerranéenne, a encore dans ce cas dicté une solution nouvelle : on a simplement colle une petite maison derrière l'église, au lieu d'une chapelle axiale, bien alignée dans l'axe du chour. Cela existe au Québec, et nulle part ailleurs.

Un dernier exemple suffira à souligner l'originalité du a métissage o québécois, celui des chapelles de procession. Cellesci sont redevables a la forme urbaine assez rare des villages linéaires québécois, établis le long d'un unique chemin, où le rituel traditionnel de la procession - faisant le tour du village pour revenir à l'église - est géométriquement impossible. Les chapelles de procession sont un dispositif particulier inventé afin de combler cette lacune de a circonférence w : imitant les églises qu'elles accom- 
pagnent, l'une à l'est du village, l'autre à l'ouest (à Sainte-Famille de l'Île-d'Orléans, par exemple), les chapelles de procession, servaient tout simplement a baliser l'itinéraire processionnel, autrement infini (parce que linéaire).

\section{LA DIVERSITÉ DE L'OFFRE}

Tout ceci laisserait croire, bien sûr, qu'il existe une figure typique de l'église : il suffirait donc d'en voir une pour les avoir toutes vues. Rien n'est plus faux. Si des caractères communs établissent l'église québécoise, les églises du Québec se distinguent toutes, régionalement, par une diversité qui rend chacune intéressante. Entre autres, les matériaux disponibles localement (le granit, le calcaire, etc.) produisent de telles variations. Les bâtisseurs d'églises ont eux-mêmes a régionalisé $*$ l'architecture ecclésiale. Si tous les grands noms de l'architecture au Québec sont liés, à un moment ou à un autre, à la construction d'églises, il faut remarquer, justement, que le rayonnement des architectes et des constructeurs, eux-mêmes tributaires de leur époque et de leur région, a constitué des * cellules $*$ bien spécifiques. Il est d'usage, par exemple, de distinguer « l'École de Quévillon $*$, responsable de plusieurs églises de la région de Montréal, des églises que Thomas Baillairgé concevait dans l'est de la province. Ainsi, si toutes les églises sont québécoises, et s'il existe une $\alpha$ église québécoise $*$ peu de monuments, au Québec, sont autant régionalistes que celles-là.

De surcroît, chaque église est aussi spécifique parce qu'elle appartient à un moment bien précis de l'histoire, dans un contexte où peu de liens sont aussi serrés entre l'État et l'Église. Les églises sont ainsi devenues le réceptacle d'une page de l'histoire nationale et nombreux sont les événements qui en ont tiré leur ancrage matériel : "l'émeute a commencé devant l'église $\nsim$, les patriotes se sont repliés dans l'église s sont parmi les phrases qui illustrent bien ce phếnomène de représentation d'une mémoire collective profondément attachée aux bâtiments ecclésiaux.

Il y a donc beaucoup à raconter dans les églises, devant les églises, au sujet des églises et du paysage québécois. Cela d'autant plus qu'elles n'ont pas subi les guerres, qu'elles sont encore fort nombreuses et, surtout, qu'un grand nombre d'entre elles ont intentionnellement été conçues comme des monuments de leur propre histoire, de leur propre spécificité. En effet, l'Église qui, de tout temps, avait cherché à récupérer sous son manteau le domaine de l'architecture ecclésiale, a trouvé quelques candidats exceptionnels chez les prêtres qui, tel le chanoine Georges Bouillon ${ }^{17}$, sont devenus architectes pour codifier très précisément, dans la configuration formelle, la signification de l'Église et de l'église. A Longueuil, à l'Ancienne-Lorette, à TroisPistoles, par exemple, on a ainsi véritablement monumentalisế toute la spécificité de l'église québécoise, inscrit dans une codification architecturale toute son identité, tous ses caractères distincts qui s'étaient accumulés dans le temps et que chaque bâttiment, dans le paysage québécois, porte à sa manière.

$* * *$

Ce sont quelques-uns des ingrédients qui peuvent servir à expliquer les églises et à en améliorer la lecture. Il paraît en effet évident, devant l'intérêt manifeste, que l'on pourrait mettre en réseau les bâtiments et visiter les églises du Québec comme on visite les châteaux de la Loire. Le chapelet serait québécois et chacune de ses perles différente, marquée comme des plats du terroir... - par une essence régionale particulière.

Mais, comme l'invoqueront certains, les églises sont aujourd'hui peu accessibles. Elles sont surtout peu accueillantes. Si l'accessibilité des églises, pour l'heure réservée à quelques initiés, peut être résolue par une fréquentation qui en assumerait les coûts, il reste encore, effectivement, à densifier cette fréquentation, bref, à construire la mise en tourisme des églises québécoises.

A ce titre, l'on regrettera peut-être un jour d'avoir si promptement dissocié les presbytères des églises, en les vouant à des fonctions strictement privées, alors qu'une mise en tourisme orchestrée, évidemment nécessaire à la conservation du patrimoine ecclésial ${ }^{18}$, aurait pu les requérir: Cependant, dans une optique plus positive, on peut souligner quelques avenues parmi celles qu'il conviendrait d'explorer, formules consacrées ailleurs que le Québec tarde à adopter. Ainsi, cer- taines pratiques pourraient contribuer à densifier l'attrait de l'objet dont l'anonymat actuel, sur le marché de l'offre touristique, tient certainement plus du mutisme des « vendeurs $*$ que des qualités qu'il recèle ou de l'intérêt certain de ses potentiels visiteurs. Parmi ces pratiques, notons : monter au clocher, visiter la crypte, établir une boutique d'objets dans l'église (selon l'usage européen), ou un coffee shop (comme en Grande-Bretagne, surtout).

En ce qui concerne la conservation du patrimoine, que nous évoquions en avantpropos de cet article, un certain nombre de réflexions devraient bien sûr être amorcées, en amont de l'offre touristique, qui, au lieu de projeter des monuments pour spécialistes, arrimerait le potentiel monumental à la mise en tourisme, quitte à déborder des limites traditionnelles et rigides de la pure restauration, par exemple. Ainsi, on peut remarquer que toutes les églises, ici, datent apparemment de la même époque. Alors que les églises romanes en France ont l'air romanes, figées dans le temps, toutes les églises du Québec, densément utilisées au long de leur histoire, et mises au goût du jour et rénovées à cette fin, datent à toutes fins pratiques des années 1950 , où la désaffectation progressive du culte les a laissées. Peut-être n'est-il pas nécessaire qu'elles s'ornent toutes des mêmes lam-

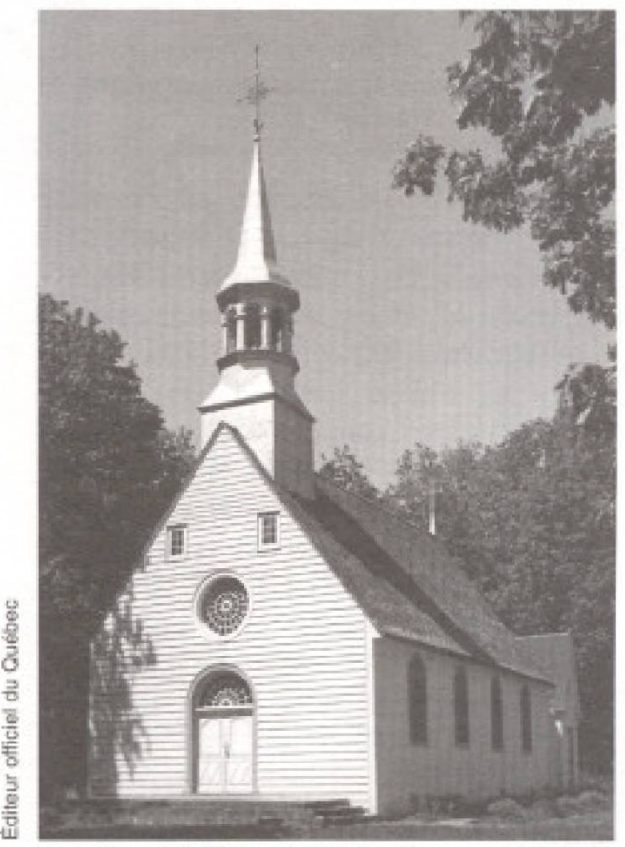

La chapelle Saint-Louis-de-Gonzague (SaintJoachim, 1780-1781) qui, tant dans ses dispositions extérieures qu'intérieures, illustre bien ce qu'était une église du Régime français. 


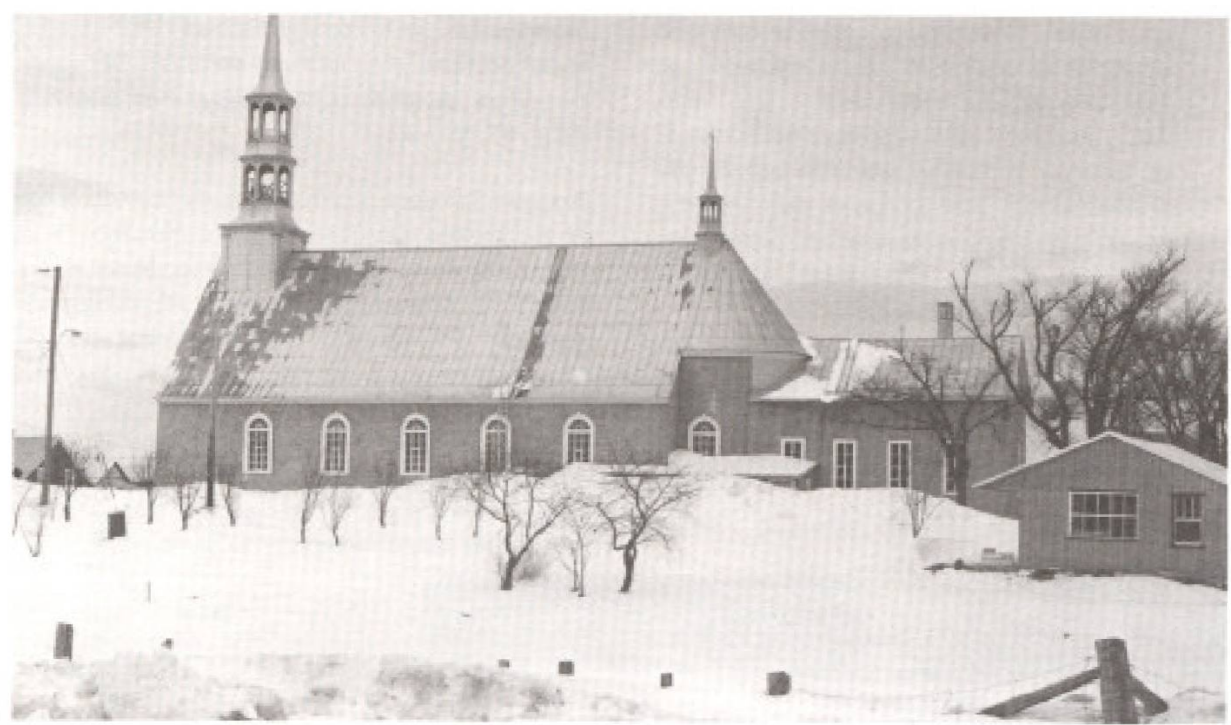

L'église Saint-André de Kamouraska et sa sacristie.

pions, de la même image de Sainte-Anne ; peut-être même serait-il bien, quelque part, de remettre en scène quelque moment passé, de densifier le message (revoir le culte sous le Régime français, par exemple). Voir les églises ainsi comme les objets d'intérêt qu'elles sont aurait aussi l'avantage de faciliter leur reconversion, si elles ne peuvent plus servir que le culte : le caractère sacré dont elles sont de toute façon investies pourrait d'ailleurs servir d'autres sacralisations - une église peut, comme on le sait, devenir panthéon, par exemple. Bref les églises, hauts lieux de l'identité du paysage québécois, peuvent continuer d'être sacrées sans continuer d'être cultuelles.

Tout cela ne concerne, dira-t-on, que le tourisme dit \& culturel $\$$ - celui qui visite les monuments et les musées. Au chapitre * tourisme et religion $*$, pourtant, de telles perspectives concernent aussi le culte. Car les églises, comme elles attireraient des touristes * ordinaires $*$, pourraient aussi attirer des croyants, par des musiques, par des décors différents ; l'expérience est d'ailleurs déjà en vogue dans quelques enclaves. Le tourisme religieux, comme le tourisme " en général \%, s'accroît évidemment du fait de l'ampleur et de la diversité de l'offre. À l'heure actuelle, sans doute rien, que les eglises, ne se prête mieux à une telle croissance.

Un comité de lecture a lu et aceepté ce texte

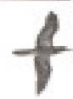

\section{NOTES}

1 Les auteurs tiemnent à remercier le Conseil de recherches en sciences humaines, le Conseil des arts et le fonds pour la formation des chercheurs et l'aide à la recherche pour le soutien apporté à leurs travaux.

2 Centre d'études interdisciplinaires sur les lettres, les arts et les traditions des francophones en Amérique du Nord.

3 Il va sans dire que, à l'instar d'un nombre croissant de chercheurs et de consultants en stratégies touristiques, nous considérons que les \& objets culturels $\approx$ (et notamment le * patrimoine $*$ ) sont à la base d'une offre touristique véritablement spécifique des diverses destinations, tout particulièrement à l'heure de la mondialisation des échanges. Voir à ce sujet, entre autres, Silberberg. 1995.

4 Il est en effet désormais admis qu'au-delà de leur vie cultuelle, les monuments ecclésiaux peuvent epouser une vie culturelle intense : nombre de ces monuments ont d'ailleurs été à l'origine même du développement de l'expertise des a monuments historiques s, en France, par exemple. En ce qui concerne le passage de la vie cultuelle à la vie culturelle, voir les articles de Mottura, 1993 : 154-157, de Mounier, 1993 : 158-160 et de Rocher, 1993 : 161166.

5 Noppen et Morisset, 1996 ; Noppen et Morisset, 1995.

6 Comme nous l'expliquons plus loin dans le texte, sous l'angle que considère notre article, en ce qui concerne le Québec, ce rapport est surtout celui entre le tourisme et les églises catholiques.
7 Sans élaborer ici sur la désaffectation des lieux de culte, il est de fait évident que les églises, de plus en plus délaissées par les assemblées de fidèles qui en assuraient autrefois l'entretien, s'offrent aujourd'hui à une reconversion touristique. D'ailleurs, dans le cas des églises auxquelles les spécialistes ont attribué le statut de * patrimoine s, il s'agit souvent de la seule avenue de conservation possible. En ce qui concerne l'avenir de ces églises, et d'autres encore anonymes, le lecteur intéressé pourra se référer notamment à Noppen et Morisset, 1997a : 7-12.

8 On pourra évidemment contester la valeur que peuvent représenter ces châteaux de la Loire au regard de toute la France ; ce n'est toutefois pas une coincidence sil un château parait en page couverture du guide Michelin La France, tel un archétype de l'offre touristique de ce pays (alors qu'une église parait en page couverture du guide Le Québec)... Les châteaux de France comptent parmi les \& destinations * les plus souvent citées par les voyageurs en partance pour la France ; selon le Conseil national du tourisme, ils arrivent d'ailleurs au deuxiẻme rang des " sites culturels les plus visités $*$ de France ( 33 millions de visiteurs par an), juste après... les édifices religieux (Monferrand, $1994: 41$ ).

9 Les églises seraient en quelque sorte, dans le paysage occidental, les flagships du paysage culturel québécois ou, pour reprendre la taxonomie élaborée il y a près de vingt ans par D. MacCannell (The Tourist : a New Theory of the Leisure Class, 1979), les églises seraient au Québec des markers et des sights. Le caractère distinctif et la notoriété de leur figure leur valent d'être à la fois des objets représentatifs du paysage qu'elles ponctuent et des attractions ₹ visitables * en soi, comme le sont les chateaux de la Loire ou le Louvre à Paris - exemples d'ailleurs invoqués par MacCannell.

10 A ce qu'il nous semble, en tous cas, et tel qu'on peut le constater par la popularité exceptionnelle des ouvrages sur les églises qui se multiplient sans cesse, ainsi que par le succès inattendu du récent Premier colloque international sur I'Avenir des biens d'Église (Québec, 5 et 6 juin 1997) - qui a accueilli plus de 300 participants, en plus de la plupart des journaux et des chaines de radio et de télévision locales et nationales.

11 Nous faisons allusion ici a l'atelier sur * l'offre touristique * organisé dans le cadre du * Fonum sur l'industrie touristique : vers une stratégie de développement économique du tourisme au Québec $\%$, Montréal, 11 mars 1997 
12 Les plus recentes publicites des organismes responsables laissent croire, th tout le moins, que le Québec n'a de richesse que ses ressources dites * naturelles $*$ et ses quelques equipements ( $*$ auberges * * péche $*$, * chasse $\%$, etc.).

13 Les exemples que nous examinons ci-après concernent souvent les paysages des villages, quoique les caractéristiques qui y sont tnumérés désignent aussi les éplises (catholiques, comme nous l'avons exposé plus haut) des centres urbains. Il va de soi que la mixité et la densité des paysages urbains tendent à amoindrir la présence de l'église qui, bien sür, prend moins d"importance dans l'offre touristique culturelle de la ville qu'elle n'en tient dans celle des sites ruraux. Nous utilisons néanmoins les exemples suivants à titre de cas d'espece, puisque les églises nous paraissent conserver, même dans la ville, cente waleur a d'offre touristique * que nous tentons ici de dégager.

14 Ces chiffres tiennent compte a la fois des eglises et des chapelles ouvertes au culte et des églises et des chapelles reconverties ou désaffectées, mais pas des bâtiments démolis. Le total dénombre les églises paroissiales catholiques, les chapelles publiques de communautés catholiques, les chapelles privées de communautés catholiques, les églises et les chapelles protestantes et les lieux de culte dits * d'autres dếnominations * (judraique, adventiste, ete.) (Noppen et Morisset, $1994: 12$ et s.)

15 Au XVIIIe síccle, les Églises dites * catholiques * (c'est-à-dire a gallicane * et * anglicane * ne différaient pas l'une de l'autre par leur culte, mais plutôt par leur association au pouvoir (français pour l'une, britannique pour l'autre). Sur les aléas de l'une et de l'autre au lendemain de la Conquête, le lecteur interessé pourra se réfërer à La présence anglicane à Ouebec : Holy Triniry Cathedral (Noppen et Morisset, $1995 ; 40-67$ ).

16 Cette particularité très originale qu'est la Loi des fabriques, il convient de le noter, correspond en fait au désir de l'Étau d'éviter le véritable * établissement * de l'Église catholique, en divisant ce qui serait ailleurs son bien foncier (et son pouvoir) pour le répartir en autant de fabriques. Chacune a lat gouverne d'un territoire restreint, la paroisse.

17 On pourra lire à ce sujet * Au Musée des Beaux-Arts du Canada : 'Une des plus belles chapelles du pays ${ }^{t} \%$ (Noppen, 1988 ; 77-98)

18 Nous ne discuterons pas ici de la nécessité culturelle et économique de la valorisation touristique du patrimoine, que nous prenons en a priori. Ceux que la question interesse pourront se référer a l'excellent texte de Davallon sur les hauts lieux (1991 : 85 . 102), de même qu"à l'ouvrage de Boniface et Fowler, Heritage and Tourism in the Global Village (1993). En ce qui concerne les églises, nous avons exploré cette question dans l'article * Le noeud gordien * (Noppen et Morisset, 1997a).

\section{BIBLIOGRAPHIE}

Anderson, Kay, ed. (1992), Inventing Places, Wiley.

Aucourt, Renê (1994), Nos Eglises, lieux de memoire de la modernite \$, Cahers Espaces: Tourisme el culture, 37, p. 151-162.

Boniface, Priscilla, et Peter J. Fowler (1993), Heritage and Tourism in the Global Village, London, Routledge.

Chaire de Tourisme (1997). Forwh de l'industrie touristique: synthese des discussions et des propasitions, Montréal, UQAM.

Davallon, Jean (1991), « Produire les hauts lieux du patrimoine s, dans André Micoud (dir), Deshauts lieux. La construction sociale de l'exemplarite, Paris, CNRS.

Monferrand, Alain (1994), * Le patrimoine culturel : un ressort méconnu du développement touristique \%, Cahiers Espace : Tourisme et culture, 37, p. 39-45.

Mottura, Pascale (1993), a Au seuil du 3e millenaire, vers un tourisme spiritualiste? $?$, Cahiers Espaces : Tourisme religien valorisation touristique du patrimoine), $30, \mathrm{p} .154-157$.

Mounier, Frédéric (1993), \& Le Mont-SaintMichel et ses magiciens *, Cahiers Espaces: Tourisme religieux (valorisation touristique da patrinoine), 30, p. 158-160.

Noppen, Lue (1988), Au Musée des Bequ-Arts du Canada: " Une des plus belles chapelles du pays \%. Ottawa, Musée des Beaux-Arts du Canada.

Noppen, Luc, et Lucie K. Morisset (1994), Lieux de culte situes sur le territoire de la ville de Québec, Québec, Ville de Québec, Division du design urbain et du patrimoine.

Noppen, Luc, et Lucie K. Morisset (1995), La présence anglicane à Québec : Holy Trinity Cathedral, Sillery, Septentrion.

Noppen, Luc, et Lucie K. Morisset (1996), Art et architecture des eglises à Quebec : * Foi et Patrie " Québec, les Publications đu Québec.
Noppen, Luc, et Lucie K. Morisset (1997a), \& Le naud gordien : réflexions en amont de l'héritage des lieux de culte 5, La revue d'architecture $A R Q, 97$, p. 7-12.

Noppen. Luc, et Lucie K. Morisset (1997b), \& De la production des monuments : paradigmes et processus de la reconnaissance $\bowtie$, Nous et les autres : la formation des espaces identitaires au Quebec, Sainte-Foy, CELAT - Presses de 1'Université Laval (a paraitre).

Rocher, Michel (1993), "IX centenaire de la Basilique de Paray-le-Monial cahiers Espaces : Tourisme religieux (valorisation touristique du patrimoine), 30, p. 161-166.

Silberberg. Ted (1995), « Cultural Tourism and Business Opportunities for Museums and Heritage Sites s, Tourism Management, août. 